\title{
Initial symptoms of nicotine dependence in adolescents
}

Joseph R DiFranza, Nancy A Rigotti, Ann D McNeill, Judith K Ockene, Judith A Savageau, Dorothy St Cyr, Mardia Coleman

\begin{abstract}
Objectives-It has been assumed that nicotine dependence has a slow onset and occurs only after prolonged daily use of tobacco. A cohort of young adolescents was followed to determine when the first symptoms of nicotine dependence occur with respect to the duration and frequency of tobacco use.

Design-A cohort of 681 seventh grade students (age 12-13 years) from seven schools in two small cities in central Massachusetts was followed over one year. Detailed information regarding tobacco use was obtained in individual confidential interviews conducted in school three times over the year. The latency time to the onset of symptoms of nicotine dependence was measured from the time a subject first smoked at a frequency of at least once per month.
\end{abstract}

Results-22\% of the 95 subjects who had initiated occasional smoking reported a symptom of nicotine dependence within four weeks of initiating monthly smoking. One or more symptoms were reported by $60(63 \%)$ of these 95 subjects. Of the 60 symptomatic subjects, $62 \%$ had reported experiencing their first symptom before smoking daily or began smoking daily only upon experiencing their first symptom.

Discussion-The first symptoms of nicotine dependence can appear within days to weeks of the onset of occasional use, often before the onset of daily smoking. The existence of three groups of individuals-rapid onset, slower onset, and resistant-distinguishable from one another by their susceptibility to nicotine dependence, is postulated.

(Tobacco Control 2000;9:313-319)

Keywords: adolescents; nicotine; addiction; dependence

Nicotine dependence is characterised by tolerance, cravings, feeling a need to use tobacco, withdrawal symptoms during periods of abstinence, and loss of control over the amount or duration of use. ${ }^{12}$ Symptoms of nicotine withdrawal include: cravings; depressed mood; irritability; frustration; anger; anxiety; difficulty concentrating; and restlessness. ${ }^{2-6} \mathrm{~A}$ popular model for the development of nicotine dependence holds that youths progress from the first cigarette through a period of occasional use and on to sustained and increasingly heavier daily use, resulting ultimately in dependence. ${ }^{7-13}$ However, it has not been established that daily use of nicotine is necessary for dependence to begin.

The assumption that heavy daily use (one half pack per day) is necessary for dependence to develop is derived from observations of "chippers", adult smokers who have not developed dependence despite smoking up to five cigarettes per day over many years. ${ }^{11} 1415$ Chippers do not differ from other smokers in their absorption and metabolism of nicotine, causing some investigators to suggest that this level of consumption may be too low to cause nicotine dependence. ${ }^{111415}$

In conflict with the assumption that prolonged daily use is a prerequisite for dependence is the observation that symptoms of nicotine dependence, which are common among adolescent smokers, appear to develop in some youths before the onset of daily smoking. ${ }^{3}{ }^{16-20}$ In a study of girls $11-17$ years of age, McNeill and colleagues were the first to find that youths report nicotine withdrawal symptoms. $^{3} \quad$ Withdrawal symptoms were reported by $74 \%$ of daily smokers and by $47 \%$ of occasional smokers. ${ }^{3}$ Other investigators have also reported withdrawal symptoms among youths who were not smoking daily at the time of the interview. ${ }^{16}{ }^{17}$ However, in these studies, individuals reporting withdrawal symptoms may have been daily smokers in the past. $^{3} 1617$ Also arguing against the need for prolonged and heavy exposure before dependence can occur is a report that $8 \%$ of subjects who had smoked 20 or fewer cigarettes over their lifetime had difficulty quitting. ${ }^{16}$

Some studies indicate that young smokers can inhale and absorb as much nicotine and carbon monoxide per cigarette as adults do, and tolerance can begin with the first dose of nicotine. ${ }^{20-23}$ Since tolerance can begin immediately, it may not be long before other symptoms of dependence follow. ${ }^{22}$ The Development and Assessment of Nicotine Dependence in Youth (DANDY) study reported here is a retrospective/prospective study of a cohort of adolescents designed to investigate the onset of symptoms of nicotine dependence. This paper presents a first look at the DANDY data, as we approach one year of longitudinal follow up.

The most commonly used definition of nicotine dependence appears in the Diagnostic and statistical manual of mental disorders, fourth revision (DSM-IV). The DSM-IV definition is based upon the assumption that "prolonged heavy use" of nicotine is required before physi- 
Table 1 Survey items concerning symptoms of dependence

1. Has the subject ever tried unsuccessfully to quit?

2. Do you smoke now because it is really hard to quit?

3. Have you ever felt like you were addicted to tobacco?

4. Do you ever have strong cravings to smoke?^

5 . Have you ever felt like you really needed a cigarette?*

6. Is it hard to keep from smoking in places where you are not supposed to, like school?

When you tried to stop smoking ... (or, when you haven't used tobacco for a while ...)

7. did you find it hard to concentrate because you couldn't smoke?

8. did you feel more irritable because you couldn't smoke?

9. did you feel a strong need or urge to smoke?

10. did you feel nervous, restless or anxious because you couldn't smoke?

11. did you feel sad, blue, or depressed because you couldn't smoke?

ॠThese items were not used as criteria for dependence because of lower specificity.

ologic dependence can occur, but acknowledges that "how quickly dependence develops is unclear". ${ }^{2}$ Since the DSM-IV definition of nicotine dependence does not allow for the possibility that dependence might start before "prolonged heavy use", the DSM-IV criteria were not used in this study. Accordingly, subjects were not diagnosed as being nicotine dependent, or experiencing a "withdrawal syndrome" according to DSM-IV criteria. Rather, we report only on whether subjects report any individual symptoms that are associated with dependence.

\section{Methods}

To study the onset of the first symptoms of nicotine dependence, a cohort of 681 seventh grade students (age 12-13 years) were enrolled in a longitudinal study. Subjects are interviewed individually in school three times each year. Four years of data collection are planned. This report presents the data from the first three interviews.

The study is being conducted in two small cities in central Massachusetts with populations of 38000 and 41000 in 1990, per capita income below the state average, and youth and adult smoking rates higher than the state average, but similar to national rates. ${ }^{24}{ }^{25}$ There were 900 seventh grade students in the seven public schools in these two cities when the study began in January 1998. The following factors contributed to the selection of these cities: their large and ethnically diverse student bodies, the cooperation of the school administrations, and student tobacco use rates comparable to national averages.

ASSEMBLY OF THE COHORT

Considerations of statistical power and anticipated attrition indicated that a minimum initial sample size of 650 would be required to allow for planned regression analyses. With the approval of the committee for the protection of human subjects in research at the University of Massachusetts Medical School, the parents of all seventh graders were sent two letters describing the study and were asked to respond if they did not want their child to participate. All students who were not eliminated by this process were assigned random numbers, and the first 650 were invited to participate. Prior tobacco use did not preclude participation. Students who declined to participate were replaced by continuing down the list of random numbers until 650 had agreed to participate. The initial 650 interviews were completed ahead of schedule, allowing the sample size to be expanded to 681 as additional students were sequentially invited to participate based upon their random number assignment. Subjects were told that the study concerned tobacco, and those who assented to participate were promised confidentiality. No subjects were added after the first set of interviews were completed in March 1998. The third interviews were completed in December 1998.

\section{SURVEY INSTRUMENT}

The survey instrument collected detailed information about prior and current tobacco use including the duration of use, the frequency of use, the amount used, the pattern of use, the types of tobacco used, periods of abstinence, and attempts to quit smoking. Students were asked to provide exact dates for the first puff, the first inhalation, the first monthly use, the first daily use, and the first occurrence of 11 symptoms of dependence (table 1). To determine how symptoms of nicotine dependence should be identified, a review of the literature was conducted to locate validated survey items used for this purpose in previous studies. ${ }^{3} 1618192627$ Twelve items were identified and pilot tested on a population of adolescents to eliminate those which produced positive responses in non-smokers (false positives). An item from the National Household Survey on Drug Abuse read "Have you ever felt addicted, or dependent on tobacco?"19 Many smokers responded "I'm not addicted, but I am dependent", meaning that they depended on tobacco to relieve stress. This item was retained without the reference to dependence. Some subjects who had never smoked responded affirmatively to the question "Have you ever felt like you really needed a cigarette?". They felt they needed to smoke to be popular. Other non-smokers reported experiencing "strong cravings" to smoke when seeing other people smoke. Items regarding craving and needing to smoke were retained in the questionnaire but were not included as symptoms of nicotine dependence for this analysis because of these false positive responses.

INTERVIEW PROTOCOL

Individual interviews were conducted in privacy in the schools. Interviewers followed a protocol but were instructed to explore positive responses to dependency symptoms in more depth. All subjects are interviewed three times annually, whether or not they have ever used tobacco. To evaluate the specificity of the survey items further, all subjects, including those who had never used tobacco, were asked questions $3-11$ in table 1 at the baseline survey. Most of the questions had to be reworded slightly to make sense to non-smokers. For example, the question "When you tried to stop smoking did you feel more irritable because you couldn't smoke?" was changed to "Do you feel more irritable when you can't smoke?".

Four techniques proven to facilitate the accurate recall of dates and events were 
employed during interviews. ${ }^{28} 29$ These included the use of "personal landmarks", "bounded recall", "decomposition", and a visual aide in the form of a personalised calendar. ${ }^{28} 29$ A calendar of significant events was created for each tobacco user, and brought to each interview to serve as a memory aide and to assist in establishing the timing and sequence of events. Specific dates for events were recorded when available. Otherwise, if an event was recalled to have occurred at the beginning of the month it was recorded as the seventh of the month, the middle of the month as the 15 th, and the end of the month as the 25th. Elapsed time was measured in completed weeks.

OUTCOME MEASURES

Subjects were considered to be tobacco users if they had ever used any form of tobacco. Subjects who had at any time smoked at least two cigarettes within a two month period were considered to be monthly smokers. Thus, the monthly smoker category could theoretically include subjects who were daily smokers from their first cigarette and subjects who had discontinued tobacco use after smoking just two cigarettes within the same week. A subject who had smoked one cigarette every other month for years would not be considered to be a monthly smoker. The onset of monthly smoking was defined as the point in time when the subject first smoked with a frequency of at least once per month. Nicotine dependence symptoms were operationally defined as follows: loss of control over the amount or duration of use as indicated by items 1 and 2 in table 1 ; an admission of feeling addicted to tobacco (item 3); difficulty controlling the behaviour as indicated by a positive response to item 6 ; or self report of any of the symptoms of nicotine withdrawal shown in table 1 (items 7-11).

Subjects were considered to have experienced an unsuccessful quit attempt if they had made a conscious decision to discontinue tobacco use but resumed use within three months. The three month cutoff was chosen to reduce the likelihood of attributing a resumption in use to dependence when it may have been caused by a change in peer group or other factors. To reduce the possibility that resumed smoking before the three month cutoff might also be caused by factors other than dependence, the interviewer inquired as to the reason for resumed smoking and made a determination as to whether the event should be counted as a relapse.

The latency to the onset of the first symptom of dependence was defined as the number of

Table 2 Cumulative experience with tobacco use at the first and third interviews.

\begin{tabular}{lll}
\hline & $\begin{array}{c}\text { At first interview, fanuary to } \\
\text { March, 1998 }(n=681)\end{array}$ & $\begin{array}{l}\text { At third interview, October to } \\
\text { December, 1998 }(n=626)\end{array}$ \\
\hline $\begin{array}{ll}\text { Tried some form of tobacco } \\
\text { Puffed on a cigarette }\end{array}$ & $205(30 \%)$ & $263(42 \%)$ \\
Puffed on a cigar & $200(29 \%)$ & $243(39 \%)$ \\
Tried smokeless tobacco & $53(8 \%)$ & $80(13 \%)$ \\
Inhaled cigarette smoke & $9(1 \%)$ & $12(2 \%)$ \\
Had smoked at least once per & $117(17 \%)$ & $156(25 \%)$ \\
$\quad$ month in two consecutive months & $75(11 \%)$ & $95(15 \%)$ \\
Had smoked daily & $28(4 \%)$ & $42(7 \%)$ \\
\hline
\end{tabular}

Categories are not mutually exclusive, subjects could be included in all rows. completed weeks passed between the initiation of monthly smoking, as defined above, and the date of the earliest presenting symptom of dependence. The first puff on a cigarette dated back to kindergarten for several subjects, and there was often a gap of several years between the first and second cigarettes. The date for the initiation of monthly smoking was therefore judged to be a superior baseline for measures of latency. Subjects reporting symptoms within the first week of monthly smoking have a latency of zero completed weeks. In the rare case of a symptom preceding monthly smoking, the latency had a negative value. Analyses were performed to determine which symptoms were the first to present. If two or more symptoms appeared on the same day, each was counted as a presenting symptom.

DATA ANALYSIS

This report reflects the status of the subjects at the completion of the third interview, which occurred 8-11 months after the first interview. The Student's $t$ test was used to compare means, and a probability value of $\mathrm{p}<0.05$ was used as a test of significance. Some subjects reported that they had experienced symptoms of dependence before the first interview. Reporting of these events might require the subject to recall information over a period of time that was greater than the four month interval between interviews during the prospective portion of the study. To test for possible recall bias, separate analyses were run to compare the results from subjects who were required to recall events over periods longer than four months (long recall) and those who were not (short recall). This also allowed us to evaluate the potential impact of repeatedly asking subjects if they had experienced symptoms of withdrawal by comparing those who reported symptoms at the first interview to those who reported them in subsequent interviews.

\section{Results}

The parents of 85 (9.4\%) of the 900 seventh grade students withheld permission for participation. Forty students (26 boys and 14 girls) declined to participate $(5.5 \%$ of the 721 invited); 39 of them were in the same school system. These refusals are attributed almost entirely to a few teachers who discouraged the participation of their students, possibly because of concern over the disruption of class time. The 681 subjects who comprise the initial cohort represent a response rate of $94.4 \%$ of the 721 students who were invited, and $75.7 \%$ of all seventh graders $(n=900)$. At entry, subjects' ages ranged from 11-15 years (mean age 12.6 years). Males represented 52\% of the study cohort and $49 \%$ of the student body. The racial and ethnic makeup of the study population, (67\% white, $20 \%$ Hispanic, 5\% African American, 5\% Asian, and 3\% other), was similar to that of the entire student body, (63\% white, 25\% Hispanic, 3\% African American, and 3\% Asian).

Subjects' cumulative experience with tobacco is presented in table 2 for the first and 
Table 3 Rates of reported symptoms of nicotine dependence at the first interview between 205 subjects who had used tobacco one or more times and 476 subjects who had not

\begin{tabular}{lll}
\hline Symptoms & Users $(n=205)$ & Non-users $(n=476)$ \\
\hline Felt like I really needed a cigarette & $19.5 \%$ & $2.5 \%$ \\
Strong urge to smoke when abstinent & $14.6 \%$ & $0.6 \%$ \\
Strong cravings to smoke & $13.2 \%$ & $1.7 \%$ \\
Feeling nervous, restless, or anxious & $12.7 \%$ & $0.6 \%$ \\
Feeling irritable & $10.7 \%$ & $0.0 \%$ \\
Difficulty concentrating & $8.3 \%$ & $0.2 \%$ \\
Felt addicted & $7.8 \%$ & $0.2 \%$ \\
Hard to refrain from smoking where it is not permitted & $6.3 \%$ & $1.1 \%$ \\
Feeling sad, blue or depressed & $5.9 \%$ & $0.8 \%$ \\
\hline
\end{tabular}

${ }^{\star}$ These items were not used as criteria for dependence because of lower specificity.

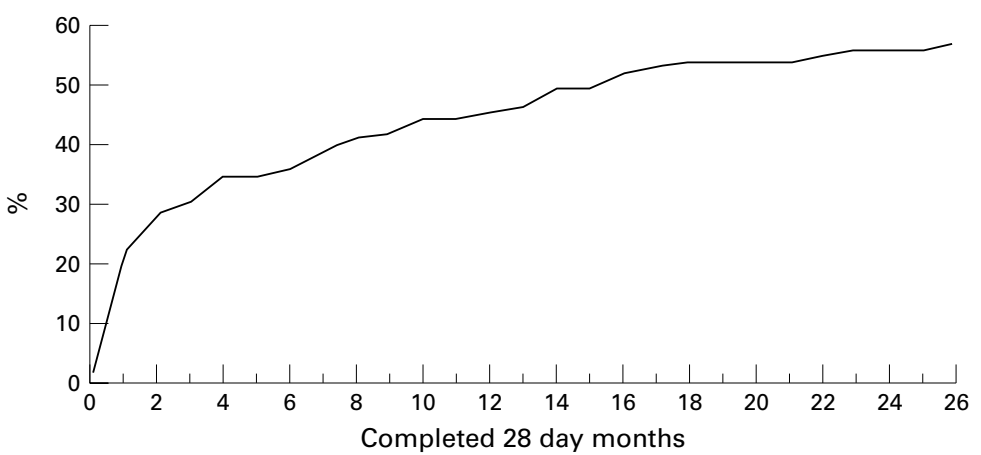

Figure 1 Cumulative incidence of the first reported symptom of nicotine dependence among 95 subjects who had ever smoked at least one cigarette in two consecutive months.

third interviews. There were no regular users of cigars or smokeless tobacco. By the third interview, conducted in October through December of 1998, subjects had moved from seventh to eighth grade and 55 subjects $(8 \%)$ had been lost to follow up, almost entirely as a result of moving out of the area. Compared to those who remained in the study, subjects who were lost were more likely to have tried cigarettes $(41 \%$ v 27\%) and to have mothers who currently smoked $(53 \% v 33 \%)$ and fathers who currently smoked ( $51 \%$ v $37 \%)$.

Table 3 compares self reported symptoms of dependence at baseline for 205 subjects who had ever tried tobacco and 476 subjects who had not. Positive responses to the dependency questions among non-users were rare with all items, but were most frequent concerning cravings and needing to smoke, items which had been excluded by design. Each symptom of dependence also was denied by the vast majority of tobacco users.

Symptoms of dependence were examined in the 95 subjects who had reported smoking monthly by the third interview and had completed all three interviews (table 4 ). The monthly smoker category includes 42 subjects who had smoked daily and 25 (26\%) former smokers. Sixty (63\%) subjects reported having experienced one or more of the nine symptoms listed in table 4 (range 1-9). Feeling addicted was the most common initial symptom, while feeling strong urges, irritable, nervous, restless or anxious when unable to smoke were the symptoms most commonly reported overall. Of the 60 symptomatic subjects, 37 (62\%) had experienced their first symptom before smoking daily or began smoking daily upon experiencing their first symptom. Of the 42 subjects who had smoked daily, six (14\%) denied all symptoms of dependence, 12 (29\%) had experienced one or more symptoms before-or simultaneous with-daily smoking, and $24(57 \%)$ experienced symptoms some time after the onset of daily smoking.

The time elapsed from the onset of monthly smoking, as defined above, to the first symptom of dependence is plotted in fig 1 . The percentages are based upon the 95 subjects who had ever smoked monthly, including 35 subjects who had not experienced symptoms. Nearly one quarter (22\%) of all monthly smokers (21/95) had reported symptoms by the end of the first month. Sixteen subjects reported symptoms within two weeks of the onset of monthly smoking, representing $25 \%$ of the 60 subjects reporting symptoms (median 14.5 weeks, mean (SD) 35.3 (48.7) weeks).

Since subjects were asked if they smoked now because it is really hard to quit, the date for this event was the date of the interview and not when the subject first failed in an attempt to quit smoking. For each of the remaining eight symptoms, one or more subjects reported experiencing the symptom within two weeks of the onset of monthly smoking (table 4). The median number of weeks to the onset of symptoms is necessarily computed based only on those subjects who have reported a symptom, and column 4 of table 4 should be interpreted accordingly.

The potential role of recall bias was assessed by comparing the responses of subjects who reported the onset of symptoms more than four months before the first interview (long recall) with those of subjects reporting symptoms after that date (short recall). Subjects with a long recall reported a slightly longer latency (mean 38.4 weeks) than those with a short recall (mean 33.1 weeks), but the difference was not significant $(\mathrm{p}=0.7)$.

Table 4 Reported symptoms associated with nicotine dependence by the third interview among 95 subjects who had at any time smoked at least once per month, including 42 subjects who had progressed to daily smoking

\begin{tabular}{|c|c|c|c|c|}
\hline Symptoms & $\begin{array}{l}\text { Number of subjects } \\
\text { reporting this as } \\
\text { their first symptom, } \\
n=95(\%)\end{array}$ & $\begin{array}{l}\text { Total number of subjects } \\
\text { reporting this symptom, } \\
n=95(\%)\end{array}$ & $\begin{array}{l}\text { Range of completed weeks from } \\
\text { the onset of monthly smoking to } \\
\text { the onset of the first symptom, } \\
n=60\end{array}$ & $\begin{array}{l}\text { Median number of weeks from } \\
\text { the onset of monthly smoking to } \\
\text { the first symptom among } 60 \\
\text { subjects reporting symptoms }\end{array}$ \\
\hline Felt addicted & $18(19)$ & $27(28)$ & -39 to 202 & 18 \\
\hline Strong urge to smoke & $14(15)$ & $37(39)$ & 1 to 199 & 28 \\
\hline Feeling nervous, restless, or anxious & $14(15)$ & $38(40)$ & 1 to 202 & 46 \\
\hline Difficulty concentrating & $13(14)$ & $30(32)$ & 2 to 209 & 32.5 \\
\hline Feeling irritable & $11(12)$ & $38(40)$ & -19 to 202 & 38 \\
\hline Unsuccessful quit attempt & $10(11)$ & $27(28)$ & -4 to 200 & 38 \\
\hline Hard to refrain from smoking where it is not permitted & $6(6)$ & $20(21)$ & 0 to 202 & 33 \\
\hline Feeling sad, blue or depressed & $6(6)$ & $17(18)$ & 1 to 202 & 30 \\
\hline Smoke now because it is really hard to quit & $0(0)$ & $16(17)$ & 17 to 228 & 72.5 \\
\hline
\end{tabular}




\section{Discussion}

This study followed a cohort of young adolescents to observe the development of symptoms associated with nicotine dependence. A quarter of the subjects who reported one or more symptoms of nicotine dependence reported experiencing their first symptom within two weeks of the onset of monthly smoking as defined above. Several subjects reported symptoms within days of starting. Is this plausible? Nicotine causes an increase in the number of high affinity nicotinic cholinergic receptors in the brain structures associated with the reward pathway in both humans and rodents. ${ }^{30-33}$ The number of these receptors increases after the second dose of nicotine. ${ }^{31}$ The increase parallels the development of tolerance, and receptor numbers decline after the drug is stopped coinciding with the withdrawal syndrome. ${ }^{30} 33$ That these high affinity receptors might play a role in dependence is also suggested by experiments with genetically altered mice. ${ }^{34}$ Mice which lack the high affinity nicotinic cholinergic receptor will not self administer nicotine. ${ }^{34}$ Nicotine infusions result in maximal up-regulation of receptors in just four days in mice, and in 10 days in rats. ${ }^{32}{ }^{33}$ The time course for up-regulation in humans has not been determined. The up-regulation of nicotinic receptors has not been established as the mechanism causing nicotine dependence, but the rapidity with which these changes in brain structure appear makes it plausible that the first symptoms of dependence might also appear rapidly.

As fig 1 demonstrates, subjects who reported experiencing a rapid onset of symptoms of dependence are not outliers, but represent a sizeable proportion of all smokers and nearly half of those who have reported symptoms thus far. In this study, symptoms of nicotine dependence were reported to be present in many smokers before daily smoking. These results are consistent with previous reports, ${ }^{31617}$ and indicate that daily smoking is unlikely therefore to be a prerequisite for the development of nicotine dependence. Subjects who have never smoked daily can fail in their cessation attempts.

These data contradict commonly held beliefs and the tendency might be to attribute them to methodological problems. The symptoms assessed in this study are subjective and were assessed through self report. Self reports of withdrawal symptoms by adolescents have shown good correlation with scores on the modified Fagerstrom Tolerance Questionnaire. ${ }^{35}$ The validity of self reports of nicotine withdrawal symptoms in adults have been established by independent observer rating and salivary cotinine concentrations. ${ }^{1}{ }^{17}$

Some have postulated that youths' experience of withdrawal symptoms may be influenced by their expectations. ${ }^{36}$ This raises the question as to whether our repeated inquiries regarding symptoms of dependence may have prompted youths to over report symptoms in subsequent interviews. There was no significant difference in the rapidity of onset of symptoms among those who had reported symptoms during the first interview and those who reported symptoms only after repeated interviews. This makes it unlikely that our results are due to repeated prompting.

Each of the items used as criteria in this study had been validated to some extent and used extensively to assess nicotine dependence in prior studies. . $^{31618192627}$ The specificity of the items used were tested by administering them to non-smokers, and items that demonstrated greater than $1 \%$ false positives after rounding were excluded. Since symptoms associated with nicotine withdrawal, such as irritability, can have other causes these symptoms were counted only if subjects attributed them to nicotine withdrawal. Since a rapid onset was documented for each of eight symptoms, all eight survey questions would have to be defective to alter the conclusion that dependence can begin rapidly. The possibility that the early onset of symptoms was an artifact of recall bias from the retrospective portion of the study was ruled out.

The use of biochemical measures of nicotine intake were considered, but not employed, because such measures cannot reliably differentiate between non-smokers and the occasional smokers who are the primary focus of this study. ${ }^{17}{ }^{21}$ This is an area for future research.

One limitation of this study is the relatively small number of subjects (60) reporting symptoms of dependence thus far. Another limitation is the narrow age range of our subjects; it is possible that the time course for the onset of dependence might be different in subjects who initiate tobacco use at different ages. Among rats, adolescents are more sensitive than adults to some of the effects of nicotine. ${ }^{37}{ }^{38}$ Human adolescents may also be more sensitive to nicotine's effects. Individuals who initiate tobacco use during early adolescence are more likely to become dependent, have more difficulty quitting, smoke for a greater number of years, and smoke more heavily. ${ }^{39-42}$ It is clear that adolescents and adults experience the same type of nicotine withdrawal symptoms, but we do not know if the intensity of the symptoms and the ability to cope with them differs between these groups. More research is needed to sort out these issues.

Based upon the data presented here, we offer a model describing three groups of individuals distinguished by their susceptibility to becoming dependent on nicotine. The rapid onset group would be those who develop symptoms of dependence within days or weeks of initiating monthly use. Several of our subjects seemed to describe a phenomenon akin to "love at first sight", sensing immediately that nicotine had a powerful influence on them.

A second group is composed of individuals who experience a slower onset to the development of symptoms of dependence. These individuals may require a more prolonged exposure to nicotine, at higher dosages, before dependence begins. Included in this group would be those individuals who do 
not report symptoms until they have been smoking for a few years. Elucidation of the physiological or psychological basis for the observed differences in the speed of onset may make it possible to establish a cut point between the rapid onset and slower onset groups.

A third group represents individuals who are particularly resistant to developing dependence. Chippers-adults who smoke up to five cigarettes per day over many years with no evidence of dependence-would fall into this group. ${ }^{11}{ }^{14}$ Our data suggest that the concentrations of nicotine to which chippers are exposed are more than adequate to cause dependence. It is too early to identify such individuals in our study, for we do not know how long a person would have to smoke without symptoms of dependence before it could be concluded that the risk of future dependence is minimal.

Within species, individual humans and genetically distinct strains of animals can differ greatly in their responses to the effects of nicotine. ${ }^{43}$ Animal studies provide biological plausibility for a model of genetically determined differences in individual susceptibility to nicotine dependence. ${ }^{45}{ }^{46}$ Our data suggest that the latency to the onset of dependence might represent a useful phenotypic trait to study in future genetic research.

The use of the term "experimenters" to refer to all less-than-daily smokers should be re-examined given the proportion of these individuals who already display symptoms of dependence. ${ }^{47}$

The DANDY study will continue with an examination of the pattern of smoking and the quantities consumed at the onset of the first symptom of dependence. The presence of one symptom does not meet the diagnostic criteria for nicotine dependence offered in DSM-IV, ${ }^{2}$ although the age of onset of nicotine dependence is defined as the age at which the first symptom of dependence occurred. ${ }^{48} \mathrm{Nico}-$ tine dependence typically begins as a paediatric condition, yet current definitions are based upon the study of adults. For example, the boundary model of nicotine dependence incorporates the observation that adult smokers experience physiologic withdrawal symptoms when daily intake falls below 8-12 cigarettes and have considerable difficulty maintaining intake below this level. ${ }^{49}$ While this model is a useful conceptualisation of dependence in adults, it failed to predict that youths would have symptoms of physical dependence before ever smoking daily. Nicotine dependence may have different manifestations in youths and adults, and current definitions of nicotine dependence need to be re-examined, especially in regard to their applicability to youths.

This study was funded by grant number CA77067-03 from the National Cancer Institute. The opinions expressed in this paper are those of the authors and do not necessarily represent the official views of the National Cancer Institute.

1 US Department of Health and Human Services. The health consequences of smoking: nicotine addiction. A report of the consequences of smoking: nicotine addiction. A report of the
Surgeon General, 1988. Rockville, Maryland: Public Health Service, Centers for Disease Control, Office on Smoking Service, Centers for Disease Control, Office on Smoking
and Health, 1988. (DHHS Publication No (CDC) a8-8406.)
2 American Psychiatric Association: Diagnostic and statistical manual of mental disorders: DSM-IV, 4th ed. Washington, manual of mental disorders: DSM-IV, 4th ed.

3 McNeill AD, West R, Jarvis M, Jackson P, Bryant A. Cigarette withdrawal symptoms in adolescent smokers. Psychopharmacology 1986;90:533-6.

4 Hatsukami DK, Hughes JR, Pickens RW, Svikis D. Tobacco withdrawal symptoms: an experimental analysis. Psychopharmacology 1984;84:231-6.

5 Hatsukami DK, Gust SW, Keenan RM. Physiologic and subjective changes from smokeless tobacco withdrawal. Clin Pharmacol Ther 1987;41:103-7.

6 Hughes JR, Hatsukami D. Signs and symptoms of tobacco withdrawal. Arch Gen Psychiatry 1986;43:289-94.

7 Jackson C. Cognitive susceptibility to smoking and initiation of smoking during childhood: a longitudinal study. Prev Med 1998;27:129-34.

8 Lamkin L, Houston TP. Nicotine dependency and adolescents: preventing and treating. Adolescent Medicine. 1998; 25:123-35.

9 Chassin L, Presson CC, Sherman SJ, Edwards DA. The natural history of cigarette smoking: Predicting youngadult smoking outcomes from adolescent smoking patterns. Health Psychol 1990;9:701-16.

10 Evans NJ, Gilpin E, Pierce JP, et al. Occasional smoking among adults: evidence from the California Tobacco Survey. Tobacco Control 1992;1:169-75.

11 Shiffman S. Refining models of dependence: variations across persons and situations. Br f Addiction 1991;86:61115.

12 Hennrikus DJ, Jeffery RW, Lando HA. Occasional smoking in a Minnesota working population. Am f Public Health 1996;86:1260-6.

13 Leventhal H, Cleary PD. The smoking problem: a review of the research and theory in behavioral risk modification. Psychol Bull 1980;88:370-405.

14 Shiffman S, Fischer LB, Zettler-Segal M, Benowitz NL. Nicotine exposure among non dependent smokers. Arch Gen Psychiatry 1990;47:333-6.

15 Brauer LH, Hatsukami D, Hanson K, Shiffman S. Smoking topography in tobacco chippers and dependent smokers. Addict Behav 1996;21:233-8

16 Barker D. Reasons for tobacco use and symptoms of nicotine withdrawal among adolescent and young adult tobacco users-United States, 1993. MMWR Morb Mortal Wkly Rep 1994;43:745-50.

17 Goddard E. Why children start smoking. Office of population censuses and surveys. London: HMSO, 1990

18 Dappen A, Schwartz RH, O'Donnell. A survey of adolescent smoking patterns. f Am Board Fam Pract 1996; 9:7-13.

19 Henningfield JE, Clayton R, Pollin W. Involvement of tobacco in alcoholism and illicit drug use. Br $\mathcal{F}$ Addiction 1990;85:279-92.

20 McNeill AD, Jarvis MJ, Stapleton JA, West RJ, Bryant A. Nicotine intake in young smokers: longitudinal study of saliva cotinine concentrations. Am $\mathcal{f}$ Public Health 1989;79:172-5

21 McNeill AD, Jarvis MJ, West R, Russell MAH, Bryant A. Saliva cotinine as an indicator of cigarette smoking in adolescents. Br 7 Addiction 1987;82:1355-60.

22 Benowitz NL. Pharmacologic aspects of cigarette smoking and nicotine addiction. N Engl f Med 1988;17:1318-30.

23 Moolchan ET, Giannas MA, Robinson ML, Pickworth WB. Smoking topography in tobacco dependent adolescents. Abstract PO 2-11 presented at the sixth annual meeting of the Society for Research on Nicotine and Tobacco February, 2000 .

24 US Department of Health and Human Services. Massachusetts and United States. Maryland: Centers for Disease Control.

25 Apt Associates. Independent evaluation of the Massachusetts Tobacco Control Program. Fanuary 1994 to fune 1995. Cambridge, Massachusetts: Apt Associates.

26 Henningfield J. Symptoms of substance dependence associated with use of cigarettes, alcohol, and illicit drugsUnited States, 1991-1992 MMWR Morb Mortal Wkly Rep 1995; 44:830-1/837-9.

27 Gfroerer J. Indicators of nicotine addiction among womenUnited States, 1991-1992. MMWR Morbo Mortal Wkly Rep 1995;44:102-5.

28 Bradburn NM, Rips LJ, Shevell SK. Answering demographic questions: the impact of memory and inference on surveys. Science 1987;236:157-61.

29 Ershler J, Leventhal H, Fleming R, Glynn K. The quitting experience for smokers in sixth through twelfth grades. $9 ; 14: 365-78$

30 Benwell ME, Balfour DJK, Anderson JM. Evidence that tobacco smoking increases the density of $(-)-\left[{ }^{3} \mathrm{H}\right]$ nicotine binding sites in human brain. I Neurochemistry 1988; 50:1243-7.

31 Ksir C, Hakan R, Hall DP, Kellar KJ. Exposure to nicotine enhances the behavioral stimulanteffect of nicotine and increases binding of $\left[{ }^{3} \mathrm{H}\right]$ acetylcholine to nicotinic receptors. Neuropharmacology 1985;24:527-31.

32 Marks MJ, Stitzel JA, Collins AC. Time course study of the effects of chronic nicotine infusion on drug response and brain receptors. F Pharmacol Exp Ther 1985;235:619-28.

33 Schwartz RD, Kellar KJ. In vivo regulation of $\left[{ }^{3} \mathrm{H}\right]$ acetylcholine recognition sites in brain by nicotinic cholinergic drugs. F Neurochemistry 1985:45;427-33. 
34 Picciotto MR, Caldarone BJ, King SL, Zachariou V, Changeux JP, Zoli M. Molecular mechanisms relevant to psychosis and nicotine dependence: use of transgenic mouse model to explore the biology of nicotinic receptors. Abstract SYM 3A presented at the sixth annual meeting of
the Society for Research on Nicotine and Tobacco. Februthe Society

35 Rojas NL, Killen JD, Haydel KF, Robinson TN. Nicotine dependence among adolescent smokers. Arch Pediatr Adolesc Med 1998;152:151-6.

36 McNeill AD, Jarvis M, West R. Subjective effects of cigarette smoking in adolescents. Psychopharmacology 1987;92:11517.

37 Faraday MM, Elliott BM, Grunberg NE. Nicotine's behavioral actions differ in adult vs, adolescent rats. Abstract PO 3-16 presented at the sixth annual meeting of the Society for Resert

38 Elliott BM, Faraday MM, Grunberg NE. Nicotine's effects on feeding and body weight differ in adolescent vs. adult on feeding and body weight differ in adolescent vs. adult sixth annual meeting of the Society for Research on Nicosixth annual meeting of the Society

39 Breslau N, Fenn N, Peterson EL. Early smoking initiation and nicotine dependence in a cohort of young adults. Drug Alcohol Depend 1993;33:129-37.

40 Breslau N, Peterson EL. Smoking cessation in young adults: age at initiation of cigarette smoking and other suspected influences. Am F Public Health 1996;86:214-20.

41 Ershler J, Leventhal H, Fleming R, Glynn K. The quitting experience for smokers in sixth through twelfth grades. Addict Behav 1989;14:365-78.
42 Taioli E, Wynder EL. Effect of the age at which smoking begins on frequency of smoking in adulthood. $N$ Engl $\mathcal{F}$ Med 1991;325:968-9.

43 Sirevaag EJ, Eisen SA, Madden PAF, Rohrbaugh JW, Heath AC. Responses to low doses of nicotine in 50 nonsmokers. Abstract PO 3-63 presented at the sixth annual meeting of the Society for Research on Nicotine and Tobacco. February, 2000.

44 Benowitz NL, Jacob P III, Jones RT, Rosenberg J. Interindividual variability in the metabolism and cardiovascular effects of nicotine in man. I Pharmacol Exp Ther 1982;221:368-72.

45 Lerman C. Caporaso NE, Audrain J, et al. Evidence suggesting the role of specific genetic factors in cigarette smoking. Health Psychology 1999;18:14-20.

46 Pomerleau OF. Individual differences in sensitivity to nicotine: implications for genetic research on nicotine dependence. Behav Genet 1995;25:161-77.

47 Friedman LS, Lichtenstein E. Smoking onset among teens: an empirical analysis of initial situations. Addict Behav 1985;10:1-13.

48 Robins LN, Helzer JE, Cottler L, Golding E. NIMH diagnostic interview schedule, version III, revised. St Louis, Missouri: Washington University; 1989.

49 Kozlowski LT, Herman CP. The interaction of psychosocial and biological determinants of tobacco use: more on the boundary model. Fournal of Applied Social Psychology 1984;14:244-56. 